\title{
Fractional Order Modeling and Control of Depth System of AUV
}

\author{
A Case Study
}

\author{
Mrs.Sneha D. Joshi \\ Department of Electrical Engineering \\ College of Engineering Pune \\ Pune, India \\ s.d.joshi@hotmail.com
}

\author{
Dr. D. B. Talange, Professor \\ Department of Electrical Engineering \\ College of Engineering Pune \\ Pune, India \\ dbt.elec@coep.ac.in
}

\begin{abstract}
In this paper Fractional Order Proportional Integral Derivative Controller (FOPID) is designed for Depth system of Autonomous Underwater Vehicle (AUV). For real time and actual implementation fractional order PID Controller is approximated. Numerous simulation comparisons are mentioned in this paper. This indicates that fractional order PID Controller if properly designed and implemented will definitely perform better than conventional integer order PID Controller.
\end{abstract}

Keywords- AUV, Fractional order calculus, Fractional Order Control, PID Control, IOPID, FOPID.

\section{INTRODUCTION}

Fractional Calculus is three centuries older like ordinary calculus [1]. Last few years apart from mathematics it is applied to several fields of engineering and technology. Fractional order system is nothing but non integer system. [2][3]

For closed loop control system there are four alternatives:-

1) Integer Order(IO) system with IO Controller.

2) IO system with FO Controller.

3) FO system with IO Controller.

4) FO system with FO Controller.

In this paper FOPID Controller and IOPID Controller are discussed for Fractional order Depth system of AUV. To design robust controller for fractional order system rather than integer order controller fractional order controller gives greater flexibility.

Nowadays, in various systems fractional order controllers are used. Software tools in MATLAB such as FOMCON, NINTEGER, CRONEL etc are available for fractional order modeling and control of dynamic systems [4] [5]. In this article these tools are used for testing and simulation of fractional order depth system of AUV.

There are various examples found in references for fractional order PID Controller. The step response characteristics are shown with iso damping property. The main contributions of this paper are:

1) Fractional calculus in modeling of dynamic systems.

2) Simulation study of fractional order model with integer order controller.

3) FOPID Controller can best perform than IO Controller.
The paper is organized as follows. In section-II AUV depth system is discussed in detail. The mathematical model of depth system and related parameters are mentioned. In section-III FOPID and basics of mathematics in FOPID are explained. In section-IV fractional order AUV Model with IOPID and FOPID Controllers is illustrated and compared. In section $-\mathrm{V}$ conclusion and future scope are discussed from the results.

\section{DEPTH SYSTEM OF AUV}

In many applications in shipping, mining, defense etc. Autonomous Underwater Vehicles are used. Modeling and control of AUV is big challenge for researchers due to its complexity and involved hydrodynamics. The complete mathematical model of AUV is divided into three subsystems:

i) Surge system

ii) Speed system

iii) Depth system.

Here depth system of AUV is considered. From the statics and dynamics of AUV linear and angular motion equations are obtained. Based on Newton's law two basic equations are used for modeling of AUV. Refer equations (1) \& (2).

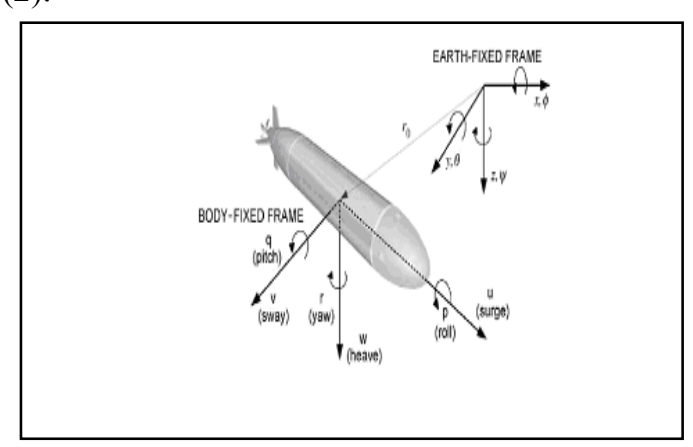

Fig.1. AUV showing all variables [6]

$$
\dot{\eta}=J(\eta) v
$$

$$
M \dot{v}+C(v) v+D(v) v+g(\eta)=\tau
$$


$J(\eta)$ - Transformation Matrix

$M$ - Inertial Matrix

$C(v)$ - Coriolis Matrix

$D(v)$ - Damping Matrix

$\eta$-Position and Orientation Vector

$\mathrm{g}(\eta)$-Gravitational Matrix

$\tau-$ Control Input Matrix

From equations (1) and (2) linear zed depth model in state space system is as follows (3): [7]

$$
\begin{aligned}
& \left(\begin{array}{cccc}
m-x_{\dot{u}} & -\left(m x_{g}+z_{g}\right) & 0 & 0 \\
-\left(m x_{g}+M_{\dot{w}}\right) & I_{y y}-M_{\dot{q}} & 0 & 0 \\
0 & 0 & 1 & 0 \\
0 & 0 & 0 & 1
\end{array}\right)\left(\begin{array}{c}
\dot{w} \\
\dot{q} \\
\dot{z} \\
\dot{\theta}
\end{array}\right)- \\
& \left(\begin{array}{cccc}
Z_{w} & m U+Z_{g} & 0 & 0 \\
M_{w} & -m x_{g} U+M_{\dot{q}} & 0 & M_{\theta} \\
1 & 0 & 0 & -U \\
0 & 1 & 0 & 0
\end{array}\right)\left(\begin{array}{c}
w \\
q \\
z \\
\theta
\end{array}\right)=\left(\begin{array}{c}
Z_{\delta_{S}} \\
M_{\delta_{S}} \\
0 \\
0
\end{array}\right)\left(\delta_{S}\right)
\end{aligned}
$$

Considering the state vector as $X$ and control vector as $u$ (4) is:

$$
x=\left(\begin{array}{llll}
w & q & z & \theta
\end{array}\right)^{T}, u=\left(\delta_{S}\right)^{T}
$$

The state space system is typically expressed as $\dot{x}=A x+B u$ With a forward velocity of vehicle as $\mathrm{U}=$ 1.54 Knots. The experimental parameter values are as in Table 1.

Table 1: Inertia and Added Mass and Drag Coefficients

\begin{tabular}{|c|c|}
\hline Parameter & Value \\
\hline$I x x$ & $1.77 \mathrm{e}-001 \mathrm{~kg} \cdot \mathrm{m}^{2}$ \\
\hline$I_{y y}$ & $3.45 \mathrm{e}+000 \mathrm{~kg} \cdot \mathrm{m}^{2}$ \\
\hline$I z z$ & $3.45 \mathrm{e}+000 \mathrm{~kg} \cdot \mathrm{m}^{2}$ \\
\hline$Y_{\dot{v}}$ & $-35.5 \mathrm{~kg}$ \\
\hline$Y_{\dot{r}}$ & $1.93 \mathrm{~kg}$ \\
\hline$N_{\dot{v}}$ & $1.93 \mathrm{kgm}$ \\
\hline$N_{\dot{r}}$ & $-4.88 \mathrm{~kg}-\mathrm{m}^{2} / \mathrm{rad}$ \\
\hline$M_{\dot{w}}$ & $-1.93 \mathrm{e}+000 \mathrm{kgm}$ \\
\hline
\end{tabular}

\begin{tabular}{|c|c|}
\hline$M_{\dot{q}}$ & $-4.88 \mathrm{e}+000 \mathrm{kgm}^{2} / \mathrm{rad}$ \\
\hline$Z_{w}$ & $-6.66 \mathrm{e}+001 \mathrm{~kg} / \mathrm{s}$ \\
\hline$M_{w}$ & $-3.07 \mathrm{e}+001 \mathrm{~kg}-\mathrm{m} / \mathrm{s}$ \\
\hline
\end{tabular}

\section{INTRODUCTION TO FRACTIONAL ORDER PID CONTROLLER}

As per the basics of fractional calculus [8] three definitions are used for fractional differentegral i.e. Grunwald Letnikov (GL), Riemann-Liouville (RL) and the Caputo.

The GL definition is given by (5)

$$
a^{D^{\alpha}}{ }_{t} f(t)=\lim _{h \rightarrow 0} h^{-r} \sum_{j=0}^{[t-a / h]}(-1)^{j}\left(\begin{array}{l}
r \\
j
\end{array}\right) f(t-j h
$$

RL Definition is given by (6)

$$
\begin{aligned}
& { }_{a} D^{\alpha}{ }_{t} f(t)=1 / \Gamma(n-r) d^{n} / d t \int_{a}^{t} f(\tau) /(t-\tau)^{r-n+1} \\
& (\mathrm{n}-1)<\mathrm{r}<\mathrm{n} \text { and } \Gamma() \text { is Gamma function. }
\end{aligned}
$$

The Caputo definition is given by (7)

$$
{ }_{a} D^{\alpha}{ }_{t} f(t)=1 / \Gamma(r-n) \int_{a}^{t} f^{n}(\tau) /(t-\tau)^{r-n+1} d \tau
$$

$(\mathrm{n}-1)<\mathrm{r}<\mathrm{n}$

The state space form of fractional order system is expressed in Laplace Transform and the transfer function is as follows (8):

$$
\mathrm{G}(\mathrm{s})=\frac{1}{a_{n} s^{\alpha_{n}}+\ldots . .+a_{1} s^{\alpha_{1}}+a_{0} s^{\alpha}}
$$

The most common form of fractional order PID controller [9] is in the $P I^{\lambda} D^{\mu}$ form. The order of integrator is $\lambda$ and that of differentiator is $\mu$ and both are real numbers. The transfer function of such PID controller is (9):

$$
G(s)=\frac{U(s)}{E(s)}=k_{P}+k_{I} \frac{1}{s^{\lambda}}+k_{D} s^{\mu}
$$

\section{$(\lambda, \mu>0)$}

$\mathrm{E}(\mathrm{s})$ is error, $\mathrm{U}(\mathrm{s})$ is controller output and $\mathrm{G}(\mathrm{s})$ is transfer function of controller. In time domain it can be expressed as: (10) 


$$
u(t)=k_{P} e(t)+k_{I} D^{\lambda} e(t)+k_{D} D^{\mu} e(t)
$$

With $\lambda, \mu=1$ the FOPID controller will behave as classical PID controller. For dynamic systems FOPID enhances control performance. The band limit of FOPID is important. The finite dimensional approximation should be done by selecting proper frequency range. Though theoretical fractional order system is with infinite memory practically, finite memory approximation is required. Different methods like Oustaloup's method are used for finite memory approximation. [10][11]

\section{COMPARIOSON BITWEEN SIMULATIONS}

\section{A. IO PID controller for Depth system of AUV}

Here simulations are carried for fractional order Depth system of AUV for IOPID and FOPID controllers. The transfer function [12] of depth system [13] of AUV in fractional order is given as (11):

$$
\text { T.F }=1 / 0.156 s^{2.7}+0.128 s^{1.8}+0.108 s^{0.9}, \alpha=0.9
$$

For optimally adjusted PID controller in integer order the values of proportional, integral and derivative gains and other performance parameters are shown in Table 2.

Fig. 2(a) \& Fig. 2(b) shows step response and bode plots for open loop depth system of AUV respectively.

Fig. 3(a) shows unit step response for the system applied with IOPID controller and Fig. 3(b) shows Bode plots of the controlled system.

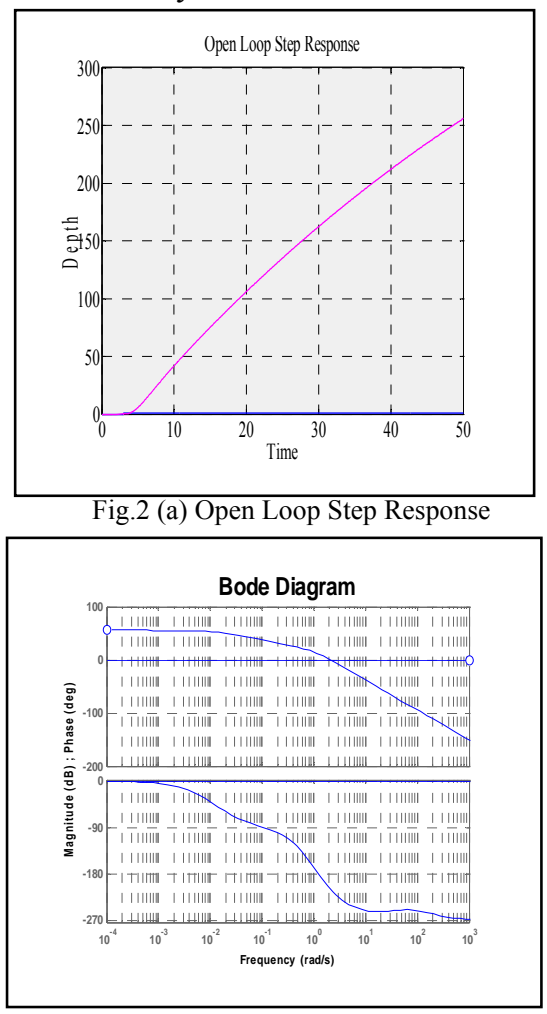

Fig. 2 (b) Bode Plots for Open Loop System

\section{B. FOPID for Depth system of AUV}

Here FOPID controller is applied to a depth system of AUV. Taking $\lambda=0.5$ and $\mu=0.6$ and with a numerical search the best

Fractional order PID controller is with $k_{p}=0.1, k_{I}=3.12, k_{D}=2.1$

Table 2: Performance Parameters IOPID

\begin{tabular}{|c|c|c|}
\hline $\begin{array}{c}\text { Sr. } \\
\text { no }\end{array}$ & Parameter & Value \\
\hline 1 & P gain & 0.03168 \\
\hline 2 & I gain & $7.4427 \mathrm{e}-005$ \\
\hline 3 & D gain & 0.268157 \\
\hline 4 & Peak Overshoot & 0 \\
\hline 5 & Rise time & 5.55 \\
\hline 6 & Settling Time & 30.3 \\
\hline 7 & Gain Margin & 16.8 \\
\hline 8 & Phase Margin & 67.4 \\
\hline
\end{tabular}

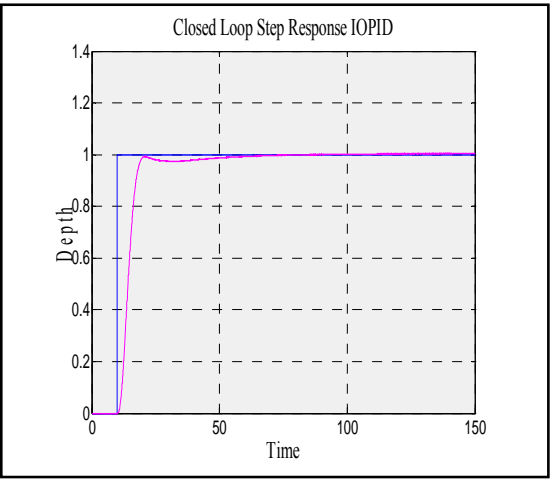

Fig.3 (a) Step Response for IOPID Controller

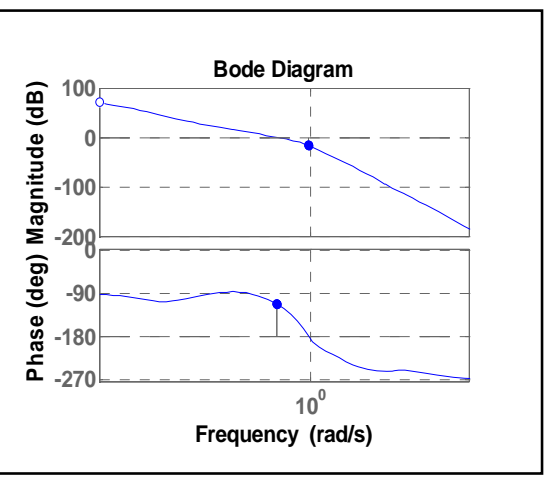

Fig. 3 (b) Bode Plots for Closed Loop System(IOPID)

The simulation block diagram is shown in Fig, 4. FOPID has two more extra parameters, $\lambda \& \mu$ than IOPID. Because of these extra parameters FOPID performs more better than IOPID [14].There is no fixed method for selection of $\lambda \& \mu$. Here the optimal values for $\lambda \& \mu$ are taken as $0.5 \& 0.6$ respectively. Similarly while implementing FOPID finite order approximation i.e. $\mathrm{N}$ has to be decided. Here two values of $\mathrm{N}$ are considered. The difference between Fig. 5(a) \& Fig.5 (b) is very small for 
$\mathrm{N}=4 \& \mathrm{~N}=5$. Bode plots for FOPID controller for fractional order depth system is as shown in Fig. 6.

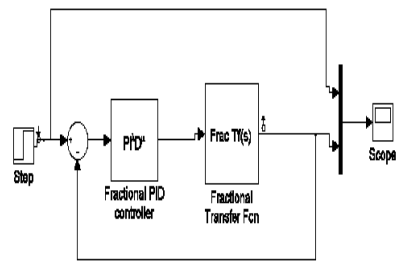

Fig, 4 Simulink Model of FOPID for FO System

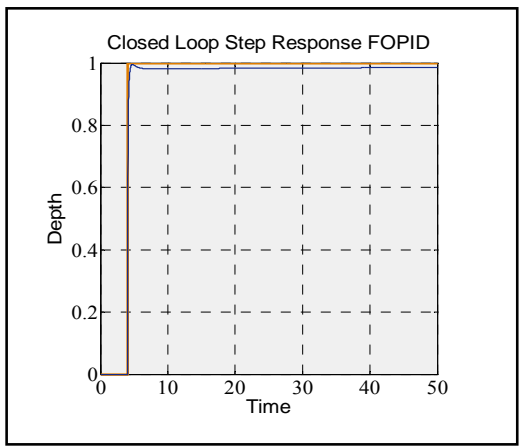

Fig.5 (a) Step Response of FOPID

$$
\mathrm{N}=4 \alpha=0.5
$$

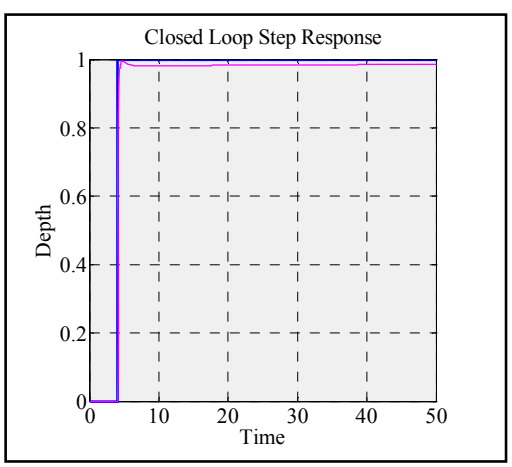

Fig.5 (b) Step Response of FOPID

$$
\mathrm{N}=5 \alpha=0.5
$$

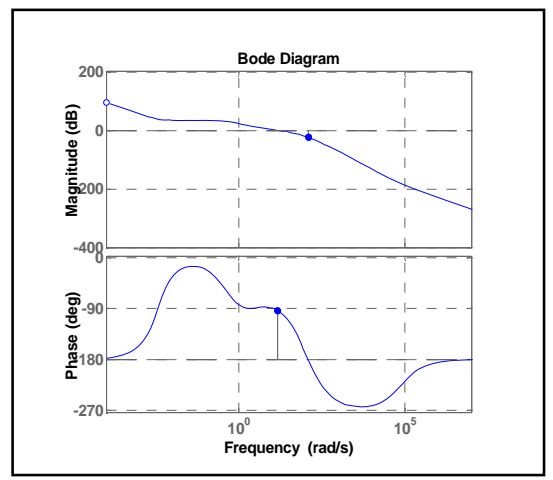

Fig.6 Bode Plots for FOPID Controller

\section{CONCLUSIONS}

In this paper a fractional order PID controller is compared with

Integer order PID for fractional order Depth system of AUV.

Using numerous simulation comparisons it indicates that the fractional order PID controller can be well implemented than ordinary integer order controller for fractional order model of depth system of AUV. FOPID works better than IOPID. From the simulation results it is seen that approximation no longer affects on performance of controller. The rise time, settling time, overshoot in FOPID are improved than IOPID. Slight steady state error is observed in the response of FOPID but is negligibly small. (Fig.5) In spite of better performance of FOPID in modeling and control aspects the research in fractional control system is still in its primary level. In future the tuning rules of FOPID may develop so also the modeling techniques.

\section{ACKNOWLEDGMENTS}

Authors wish to thank Naval Research Board (NRB) who have supported this project under their grant in scheme Projects. Project no. NRB-236/SC/11-12

\section{REFERENCES}

[1] K. B. Oldham and J. Spanier, The Fractional Calculus, Academic Press, New York and London; 1974.

[2] I.Podlubny, Fractional Differential Equations, Mathematics in Science and Engineering ,3rd ed. San Diedgo; Academic Press, 1999.

[3] Dingy"u Xue and YangQuan Chen, Advanced Mathematic Problem Solution Using MATLAB, Beijing: Tsinghua University Press; 2004.

[4] M. Benchohra, Z. Boutefal, Impulsive Differential Equations of Fractional Order with Indefinite Delay, Journal of Fractional Calculus and Applications, Vol. 5. July 2013, No. 5, pp. 1-15.

[5] Aleksei Teplijakov, Eduward Petlekov, Juri Pelikov;FOMCON Fractional Order,Modeling and Control Toolbox for MATLAB; Published in 2011

[6] Chen Yang, "Modular Modeling and Control for Autonomous Underwater Vehicle (AUV)"'Thesis of Master of Engineering Department of Mechanical Engg National Universityof Singapore 2007.

[7] Timothy Prestero, Verification of a Six-Degree of Freedom Simulation Model for the REMUS AutonomousUnderwater Vehicle, Massachusetts Institute of Technology and the WoodsHole Oceanographic Institution September 2001,M.S.Thesis.

[8] Ivo Petr'a Fractional - Order Feedback Control of A DC Motor of A DC Motor, Journal of Electrical Engineering VOL 60, NO. $3,2009,117-128$

[9] I. Podlubny, Fractional Order systems and PI $\alpha \mathrm{D} \lambda$ controllers, IEEE Transactions on Automatic control.vol.44,no.1 pp208-214,1999.

[10] R. Caponetto, L. Fortuna, and D. Porto, "A new tuning strategy for a non integer order PID controller," IFAC2004, Bordeaux, France, 2004

[11] Chunna Zhao, Dingy"u Xue and Yangquan Chen, "A fractional order PID tuning algorithm for a class of fractional order planst," Proc. Of the IEEE International Conference on Mechatronics and Automation, Niagara Falls, Canada, 2005, pp. 216-221.

[12] Lubomir Dorcak,Ivo Petras, Imrich Kostial andJanTerpak,Fractional Order State Space Models; International Carpathian Control Conference ICCC 2002 Malenovice Czech Republic,May 27-30 2002. 
[13] C.Monje,Y.Chen,B.Vinagre,D.Xue and V.Feliu Fractional Order Fystemsand
Applications,SpringerLondonDordrecht Heidelberg NewYork,2010.

[14] K. Sundaravadivu, K. SaravananDesign of Fractional Order PID Controller forLiquid Level Control of Spherical Tank, European Journal of Scientific ResearchISSN 1450-216X Vol.84 No.3 (2012), pp 345-353. 